\title{
NEW DEVELOPMENTS IN ASTEROSEISMOLOGY
}

\author{
D. W. KURTZ \\ Department of Astronomy, University of Cape Town, \\ Rondebosch 7701, South Africa
}

\section{Introduction}

The day before the start of IAU Symposium 185 Wojtek Dziembowski and I were sitting in the beautiful 300-year-old garden of Shisendo in Kyoto discussing asteroseismology. Wojtek put his fingertips together to make a little temple of his hands in front of him. Then, slowly moving this temple up and down in front of him, while staring off at the green Japanese mosses in the dappled sun and shade of Shisendo's garden forest he said to me in his studied way, "Don, I am not sure that there is any asteroseismology yet". I thought, "Uh-oh. If others believe that is true, then they may not think I have much to say in my review of new developments in asteroseismology at S185." Yet I do have much to say, so I think I had better define what I mean by asteroseismology.

Sometimes I think that between theoreticians and observers, and between helioseismologists and asteroseismologists, there is potential confusion by what we mean when we say "asteroseismology". It reminds me of the story of a Western drug company which some years ago decided to market their headache remedy, a kind of aspirin, in Japan. To avoid the problem of translating their English into correct Japanese, they decided that there would be no written description on the box containing the pills. Instead, a long, narrow box was designed on which three pictures fit side-by-side. The first picture showed a woman's face with her eyes squeezed shut with the terrible pain of a headache; sparks shot out of her head. In the middle picture the look on her face was neutral as she was seen taking two of the pills with a glass of water. Then, in the last picture, she had a beaming smile; clearly her headache was gone. Well, sales were so low as to be nearly non-existent. The Japanese were not interested in this product. And the reason is obvious, of course: The Japanese read from right to left.

Maybe the confusion between helioseismologists and asteroseismologists comes from us "reading" in opposite directions. Helioseismology has incredible depth, with millions of pulsation frequencies and modes, with the physics being pushed to precisions of fractions of a percent. Whereas for stars we have at best a few hundreds of modes (in white dwarfs) and less than a few dozen modes in any main sequence star. That is a pitifully small amount of data to claim we are doing seismology, in comparison with the sun. The paucity of the data is what stimulated Wojtek's comment to me. But with stars we have essentially an unlimited number of subjects on which to work. And the physical conditions are 
wonderfully varied. There is so much more with which to confront seismology theory than for the single star, the sun.

So here is my definition of asteroseismology: We are doing asteroseismology whenever we can constrain the physics of any star, in any way, by using two or more pulsation frequencies - at a minimum, just two frequencies; not necessarily millions as in the sun. And with that definition, I have a vast amount of exciting new work to discuss in this review of "New Developments in Asteroseismology". For details and depth I urge you to read the asteroseismology papers in these proceedings to see the excitement in the field and the excitement we experienced at the Symposium:

- Helioseismology desperately wants observations of g-modes (just one will do to start with) to probe the core of the sun. Read in Kevin Krisciunas's paper about an entire class of g-mode pulsators only slightly hotter than the sun, the $\gamma$ Dor stars.

- Find out from Conny Aerts how HIPPARCOS discovered over 100 new Slowly Pulsating B (SPB) stars, hotter g-mode pulsators, when before HIPPARCOS there were less than a dozen known. These SPB stars fill a theoretical instability strip that was predicted before the observations!

- Find out from Tim Bedding and from Christoph Keller how the search for solar-type oscillations is going in other $\mathrm{G}$ stars.

- Read below, in section 2, my opinion on the possibility of a g-mode in the famous, and now controversial, star 51 Pegasi.

- Discover from Dietrich Baade and Wojtek Dziembowski about the observation and theory of pulsation in the B stars on and above the main sequence.

- See the most exciting new result of this Symposium in the paper by Bob Stobie et al.: The discovery of 2 to 3 minute pulsations in a group of sub-dwarf B stars, the romantically-named EC 14026 stars. Very satisfyingly, read about the theoretical prediction of these pulsations in the paper by Gilles Fontaine et al., a prediction made independently of the discovery. These stars are so exciting that I cannot resist discussing them some myself in section 3 below.

- Read about the latest observational results on the rapidly oscillating Ap stars in Jaymie Matthews' review (it is too bad for you, if you missed the actual performance at S185), the latest theoretical work in the paper by Hideyuki Saio and Alfred Gautschy, and some new results from me and my colleagues in section 5 below. Especially, read how HIPPARCOS trigonometric parallaxes and our asteroseismic parallaxes agree. We are doing asteroseismology!

- The $\delta$ Scuti stars are the main sequence stars with the largest number of pulsation frequencies and modes. Read Michel Breger's observational review and Joyce Guzik's theoretical discussion.

- The white dwarfs are the current asteroseismological superstars. They have hundreds of frequencies and dozens of modes. Asteroseismic masses have a precision of a few hundredths of a solar mass. Seismological distances and the trigonometric parallax of GD358 agree: asteroseismology works. Rotational splitting is observed and studied; 
possible magnetic splitting has been seen. There even seems to be the prospect that a massive $\left(M>1 M_{\odot}\right)$ DAV (ZZ Ceti) star may show the effects of differential crystallisation of Oxygen (i.e. a change in the internal structure, heat budget, and hence pulsation frequencies caused by Oxygen "snow"). Read all about it in the papers by S185's white dwarf tag-team, Chris Clemens and Steve Kawaler.

- And don't miss the poster paper by Brian Warner and Liza van Zyl, who have discovered that GW Lib, a cataclysmic variable with an orbital period of less than 2 hours, has a ZZ Ceti primary star. This pulsating white dwarf is probably about a billion years old and should have a surface temperature of $9000 \mathrm{~K}$. But accretion heating has pumped its temperature up to $12000 \mathrm{~K}$ and pushed it into the DAV instability strip. All DAV stars have masses of about 0.6 solar masses, but GW Lib may have a very different mass, and asteroseismology will tell.

That is my conclusion to this review, given right here in the introduction: Asteroseismology is alive, well, and "asteroseismology will tell". If you were not there at Symposium 185 , if you missed this IAU GA, I hope you are feeling a tinge of regret. Assuage it by reading the rest of these proceedings.

\section{Have g-modes already been discovered in a solar analogue?}

In 1995 radial velocity variations were announced by Mayor and Queloz (1995) with an amplitude of about $56 \mathrm{~m} \mathrm{~s}^{-1}$ and a period of 4.23 days in 51 Peg. Marcy et al. (1997) independently confirmed these results. The interpretation of these radial velocity variations is that there is a planet of mass at least half that of Jupiter orbiting at a distance of $0.05 \mathrm{au}$ from a near-twin to the sun. It was widely believed prior to this discovery that "gas giant" (really liquid giant) planets could only exist far from their parent star, as they do in our solar system. The discovery of a planet of Jupiter-sized mass with a semi-major axis only $20 \%$ that of Mercury is very exciting, and led to much discussion and theoretical modelling of how such an improbable planet could form, evolve and survive.

The radial velocity variations in 51 Peg have an excellent signal-to-noise ratio. There is no dispute about their reality. After their announcement everyone believed in the planet. But then the 27 February 1997 issue of Nature hit the news-stands with a flurry of worldwide media coverage. This was because "Dolly", the cloned sheep, was the cover-girl. However, also noted on the cover of that issue, but not so widely reported in the world's newspapers, was a paper by David Gray (Gray 1997) announcing the discovery of line profile variations in $51 \mathrm{Peg}$ with the same 4.23-d period as the radial velocity variations.

The implication of this, and Gray's conclusion, is that there is no planet orbiting 51 Pegasi! The radial velocity shifts in 51 Peg caused by reflex Doppler shifts (as the purported planet orbited it every 4.23 days) should cause no change in the shape of the spectral lines, only in their wavelength. The presence of variations in the line profiles can only be explained by stellar pulsation, and a period as long as 4.23 days in a solar-type star demands a single, very high-overtone g-mode. A lot of excitement ensued. Some of the people involved with the planet hypothesis and the original discovery of the radial velocity variations were vigorously dismissive of Gray's result, even publicly dismissive of Gray himself. The critics said to Gray "Let us see your data. We do not believe your result." Thus ensued a great scientific battle - one with defenders of an improbable planet battling the proposers of an improbable g-mode. Marcy et al. (1997) say, "The only viable inter- 
pretation is a companion ..." Gray \& Hatzes (1997) say, “... the planet hypothesis is no longer viable ..." This battle is not over. Both sides, and some neutral on-lookers, are gathering more ammunition in further, time-consuming, painstaking observations.

Gray \& Hatzes (1997) (from here on G\&H) accepted the challenge to publish their data, and I have had a good look at it, so here is my opinion: G\&H measured the "bisector" in a spectral line of $\mathrm{Fe}$ in $51 \mathrm{Peg}$ at three depths, $85 \%, 71 \%$ and $48 \%$ of the continuum intensity. Spectral lines are not symmetric, the bisector in $51 \mathrm{Peg}$, typical of solar-type stars, has a characteristic " $C$ " shape. If the planet hypothesis is true for $51 \mathrm{Peg}$, then the expectation is that the radial velocity variations at all three depths will be the same, i.e. there will be no change in the bisector shape. G\&H claim to have found 4.23-d variability in the bisector shape, which means that the radial velocity variations at the three depths are not identical. A planet cannot cause this.

51 PEG LINE BISECTOR DATA

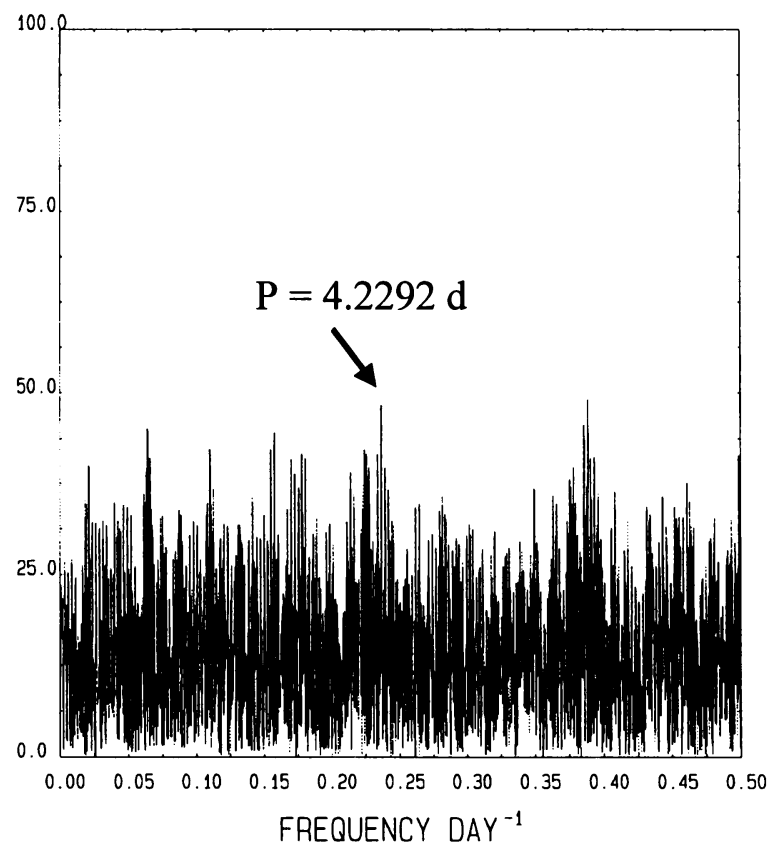

Figure 1. This is an amplitude spectrum of G\&H's line bisector data showing that the highest peak is at $\mathrm{P}=4.23$ days, and that its amplitude barely exceeds the noise. The ordinate scale is in $\mathrm{m} \mathrm{s}^{-1}$.

I have Fourier analysed G\&H's bisector data. The amplitude spectrum I get is shown in Figure 1. In the absence of any outside knowledge, I would conclude that there is no significant signal whatsoever in this amplitude spectrum. G\&H argue that the peak at $4.2292 \mathrm{~d}$ is precisely at the period of the radial velocity variations, and that the probability of finding the highest peak in the amplitude spectrum at this particular period is extremely low. They, therefore, conclude that there is real variation in the line bisector, hence the line profile. They point out that they have observed many other solar-type stars, and that the 
amplitude spectra of the bisector data for those stars show significantly lower noise than that in figure 1.

I concede that there is some merit to their argument. They may have evidence of profile variability. The reason that I am not convinced is that the amplitude spectrum shown in figure 1 is for data which consists of the difference of two signals, both of which have the full radial velocity variation (if there is no profile variation). If any systematic effect is present, then a signal in the difference could be generated as an artefact. G\&H argue that this is not probable, since the amplitude of the profile variability is nearly as large as the radial velocity variations themselves. The problem, as I see it, is that a small systematic difference might add in complex space to increase the amplitude of a random noise peak at $\mathrm{P}=4.23$ days to give, as an artefact, the peak in figure 1 . The only way to test this is with further data, and such data are being gathered.

The importance of line profile variability cannot be overemphasised. While most of the astronomical community is excited by the idea of an improbable, Jupiter-sized planet at 0.05 au from a solar-type star, we in the asteroseismology community find a g-mode of period 4.23 days even more exciting and improbable. To explain the line profile variability Gray \& Hatzes suggest a single, sectoral g-mode of low degree, but very high overtone, $n>$ 100. I have heard Paul Butler, a member of Marcy et al. (1997), publicly dismiss the pulsation hypothesis as absurd on the basis of the required $n$, and only one "observed mode". But in the roAp star HD 134214 we see a single p-mode which probably has an overtone of order $n \approx 40$. Given that, I do not "know" that a single g-mode with $n>100$ is impossible in $51 \mathrm{Peg}$. The observations will have to decide that. However, it does seem improbable, and it is extremely exciting for asteroseismology, if true.

Contrary to the antagonists' feelings in this controversy, this is a no-lose situation. Either we get an amazing planet and a new view of the formation and dynamics of planetary systems, or we get a high-overtone g-mode and the ability to probe the core of a solar-type star. New physical understanding is the result either way, thanks to the outstanding observations being produced by all groups working on $51 \mathrm{Peg}$.

\section{EC 14026 stars}

You haven't yet heard of the EC 14026 stars? Then you are guilty of not reading the important literature! Because there they are: in figure 2, reproduced right from the front cover of the Annual Report of the South African Astronomical Observatory! Just to toot the South African horn a bit louder here, I'd like to point out that in their textbook, Stellar Interiors, Hansen and Kawaler (1991) list (in their Table 2.4) thirteen classes of intrinsically variable stars. To that list the $\gamma$ Dor stars and the EC 14026 stars now need to be added. Of the fifteen classes, two of them - the EC14026 and roAp stars - were discovered at SAAO, and the seminal work of Alan Cousins on $\gamma$ Dor itself was done at SAAO.

Most of the EC 14026 stars have pulsation periods of only 2 to 3 minutes! You can read the details of the early discoveries in a series of published papers (Kilkenny et al. 1997; Koen et al. 1997; Stobie et al. 1997; O'Donoghue et al. 1997), and you can read about the independent theoretical prediction that such pulsations should be driven in Charpinet et al. (1997). You can turn right now to the review by Stobie et al. of these stars in this volume. Of particular interest, note the lower light curve in figure 2. Here is an EC14026 star in an eclipsing binary with an orbital period of 2.4 hours! In that famous textbook, Nonradial Oscillations of Stars, Unno et al. (1989) discuss (in their section 6.2) the prospect of 
determining pulsation degree, $\ell$, from the phase shift of a pulsation mode as the pulsator is eclipsed by a companion. At the time of the writing of that textbook, there were no proven cases of such a pulsator in an eclipsing binary. Now there is - the EC 14026 star you see in figure 2. We await the results of the careful modelling of the reflection effect in this system; once that is removed it will be possible to study the phase behaviour of the pulsation through ingress and egress of the primary partial eclipse, and determine the degree of the mode.

\section{South African Astronomical Observatory}
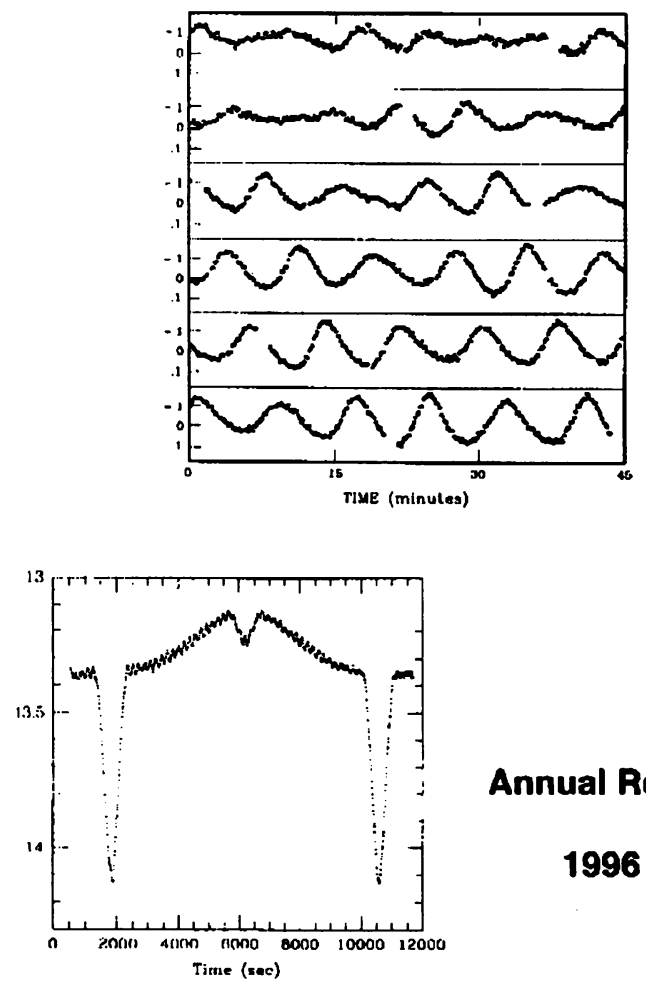

Annual Report 1996

Figure 2. The front cover of the 1996 Annual Report of the SAAO showing a 4.5-hr light curve of one of the longer period, $\mathrm{P} \approx 8 \mathrm{~min}, \mathrm{EC} 14026$ stars. The peak-to-peak amplitude is about $15 \%$. The bottom amazing light curve shows an EC 14026 star with periods near $140 \mathrm{~s}$ and $180 \mathrm{~s}$ in an eclipsing binary with an orbital period of $2.4 \mathrm{hr}$. 
I consider the discovery of the EC 14026 stars to be very important. They are sdB stars on the extreme blue end of the Horizontal Branch. The evolutionary pathway to that position in the HR diagram is not completely understood. These stars are nearly pure Helium stars with masses near $0.5 \mathrm{M}_{\odot}$, with a very thin $\mathrm{H}$ atmosphere - only about $2 \%$ of the mass of the star. This is insufficient to sustain $\mathrm{H}$ burning, so these stars run purely off of $\mathrm{He}$ fusion.

The way in which a Red Giant which is $\mathrm{H}$ shell burning undergoes a helium flash and then sheds almost all of its $\mathrm{H}$ envelope is not fully understood. There are models in which a single star is able to do that; there is another model which requires a binary system to get to the sdB state. The pulsations in the EC14026 stars can elucidate the internal structure of these stars through the techniques of asteroseismology. It may be possible to constrain, or determine, the precise thickness of the $\mathrm{H}$ layer this way. Asteroseismology of white dwarfs has successfully done this. A better understanding of the structure of the EC 14026 stars may lead to an understanding of how they have evolved to their current states.

The evolutionary time-scale for He burning has also not been tested observationally. It is possible that in a few years period changes may be measured in the EC14026 stars which are directly the result of internal structure changes caused by the on-going $\mathrm{He}$ fusion. Thus the period changes may also constrain the He fusion time-scale.

\section{Metallicism and pulsation}

Gravitational settling and radiatively driven levitation are important physical effects in many stars. Gravitational settling of helium in the sun is part of the standard solar model (Bahcall \& Pinsonneault 1992). One result is that He settling slightly ameliorates the solar neutrino problem by about $7 \%$. Metal settling may help explain the $\mathrm{Li}$ and $\mathrm{Be}$ abundances seen in the solar atmosphere. Those elements are fused at temperatures of $1-2$ million $\mathrm{K}$, and the solar model needs very fine tuning to get the convection and overshoot mixing to take the isotopes of $\mathrm{Li}$ and $\mathrm{Be}$ to just the right depth (Richard et al. 1996).

Theoretical calculations suggest that the pulsation in the EC 14026 stars is driven by the $\kappa$-mechanism operating on the iron opacity in a layer in the star in which radiative levitation has substantially increased the iron abundance (Charpinet et al. 1997). Only the combination of diffusion theory and the OPAL opacities leads to an understanding of their pulsation mechanism.

Gravitational settling of helium in Globular Cluster stars releases gravitational potential energy, heats the stellar cores, hence causes the stars to burn more brightly and have shorter lifetimes than in the absence of settling. This gives younger age determinations for the globular clusters and partially eases the discrepancy between their ages and the Hubble Age of the universe (Chaboyer et al. 1996). In even more exotic circumstances gravitational settling of helium droplets (helium rain) powers Jupiter's infra-red emission, and differential crystallisation in massive white dwarfs may result in Oxygen "snow" (see Kawaler, this volume). Gravitational settling also plays a part in the "Li problem" in F stars. Brian Chaboyer's discussion of the Li problem at this Symposium is the clearest, most thorough I have heard.

Of all the stars for which radiative diffusion (both gravitational settling and radiative levitation) is important, the stars which show the greatest effect are the chemically peculiar stars of the upper main sequence. Amongst the A stars these are primarily the nonmagnetic Am stars and the magnetic Ap stars. About 30 percent of the stars in the lower 
instability strip (where it crosses the main sequence among the A and early $\mathrm{F}$ stars) are $\delta$ Scuti stars which pulsate with amplitudes ranging from a few mmag to nearly a mag. Most of the non-pulsating stars in this part of the instability strip are spectrally peculiar mostly Am and Ap stars. Only a few Am and Ap stars pulsate, and it is a strength of diffusion theory that it can explain this. In stable A and early F star atmospheres helium settles gravitationally. This shuts off the $\kappa$-mechanism operating in the $\mathrm{He}$ II ionisation zone which drives $\delta$ Scuti pulsation. It was one time thought that a strength of the general exclusion between the pulsators and the Am and Ap stars is that it avoids the necessity of explaining how stars with surface radial velocity variations of $\mathrm{km} \mathrm{s}^{-1}$ can be stable to turbulence at the level of fractions of $\mathrm{a} \mathrm{cm} \mathrm{s}^{-1}$, which is typical of calculated diffusion velocities. However, it is now clear that this necessity cannot be avoided.

For a few stars that show abundance anomalies thought to be caused by diffusion processes, and which also pulsate, the implication is that the pulsation is laminar and generates no turbulence. These stars include some evolved Am stars near the cool border of the $\delta$ Scuti instability strip; a few marginal Am stars, and one classical Am star - all of which pulsate with amplitudes of a few hundredths of a magnitude, or less — and the rapidly oscillating Ap stars. It also includes the large amplitude peculiar star, HD 40765 . In that star Kurtz et al. (1995) conclude that $10 \mathrm{~km} \mathrm{~s}^{-1}$ pulsation generates no turbulence at the level of a fraction of $\mathrm{cm} \mathrm{s}^{-1}$.

The study of stellar pulsation (asteroseismology for stars with two or more frequencies) thus probes the atmospheric conditions under which diffusion may occur.

\section{Rapidly oscillating Ap stars}

The roAp stars are the only main sequence stars which have p-mode pulsations at all similar to those in the sun. In this volume they are reviewed observationally by the electric (literally at S185) lecturer, Jaymie Matthews, and theoretically by Hideyuki Saio \& Alfred Gautschy. I present new results from the work of my colleagues and myself here, too.

\subsection{ASTEROSEISMIC LUMINOSITIES}

The frequency spacings of 12 roAp stars allow theoretical asteroseismic estimates of their luminosities. These stars are spectroscopically so peculiar that these luminosities have been thought to be the best available. However, severe doubt was thrown on this by Dziembowski \& Goode (1996) when they calculated that the magnetic perturbation to the pulsation frequencies is so large (of order tens of $\mu \mathrm{Hz}$ ) that perturbation theory will not work, and the development of the oblique pulsator model (e. g. see Shibahashi \& Takata 1993; Takata \& Shibahashi 1995) was on shaky ground.

With the new HIPPARCOS parallaxes Matthews et al. (1997) have been able to test the asteroseismic luminosities. Figure 3 is from their paper, and it shows good agreement: Asteroseismology works. There are two exceptions. One is HD 166473 which, according to the HIPPARCOS parallax, has a luminosity only $40 \%$ that of the sun. For a main sequence A star this is obviously incorrect, so we conclude that the HIPPARCOS parallax is wrong. For $\alpha \mathrm{Cir}$ the discrepancy is a bigger problem. The asteroseismic parallax is determined from a frequency spacing of $50 \mu \mathrm{Hz}$ (Kurtz et al. 1994). The secondary frequencies in this star give a strong indication that $50 \mu \mathrm{Hz}$ is the right value, but they are 
all of amplitude about $0.2 \mathrm{mmag}$, so this needs confirmation. In this important case another intensive study of $\alpha$ Cir is called for.

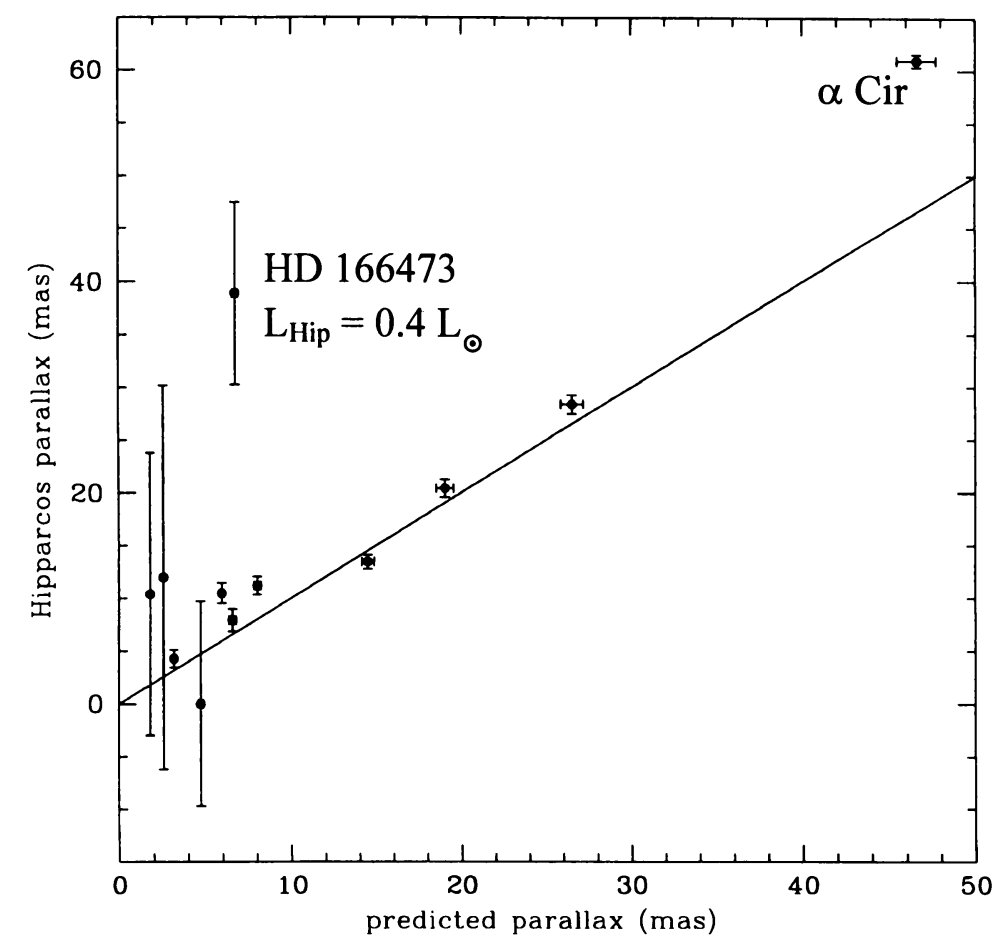

Figure 3. A comparison of the HIPPARCOS and asteroseismic parallaxes. The asteroseismic parallaxes are systematically slight smaller, and two stars (discussed in the text) stand out. Otherwise, the agreement is remarkably good - indicating that the asteroseismic luminosities are correct.

For the remainder it can be seen from Figure 3 that the asteroseismic parallaxes are systematically slightly smaller than the HIPPARCOS parallaxes. That means that the A star models used to predict the luminosity from the frequency separations systematically give too large a luminosity, hence the models are too hot and/or too large in radius, or the magnetic field affects the frequency spacings. To get an order of magnitude feel for the discrepancy: If we assume the radii are correct, then the parallax disagreement indicates that the effective temperatures of the roAp stars are about $1000 \mathrm{~K}$ cooler than the A star models from which the asteroseismic luminosities were calculated.

I remind you that the atmospheres of the roAp stars are peculiar to pathological. Luminosities are notoriously difficult to determine, and even the effective temperature can lead to decades-long, acrimonious dispute. In the most extreme case, HD 101065, temperature estimates range from less than $6000 \mathrm{~K}$ to over $8000 \mathrm{~K}$ ! This particular star is arguably the most peculiar in the sky. In its visible spectrum the lead role is played by singly ionised Holmium, with strong supporting roles from Dysprosium, Neodymium, 
Gadolinium, Samarium, Lanthanum, etc. (presuming you know where "etc." leads with that series as a starter).

Asteroseismology is providing unique constraints on the structure of the Ap stars.

\subsection{THE SIXTH FREQUENCY OF HR 1217}

In 1989 a group of 15 astronomers published an analysis of HR 1217 based on a large multi-site observing campaign (Kurtz et al. 1989). The amplitude spectrum of this star has a set of five frequencies which are separated by alternating $33.3 \mu \mathrm{Hz}$ and $34.7 \mu \mathrm{Hz}$. Shibahashi \& Saio (1985) had noted that these separations are most consistent with alternating even and odd degree modes, and that the even degree modes are most likely to be radial. But there were problems. One is that the "even" degree modes show amplitude modulation with the 12.46-day rotation period of the star. Radial modes should show no amplitude modulation with rotation; they look the same from all aspects. The other problem is that there is a sixth mode, which is separated from the fifth by $50 \mu \mathrm{Hz}$. With the interpretation of the first five frequencies as alternating even and odd $\ell$ modes, the fundamental frequency spacing implied is $68 \mu \mathrm{Hz}$. The ratio of $50 / 68=3 / 4$, and there is no known way to generate $p$-modes separated at $3 / 4 \Delta v_{0}$.

There is another interpretation. That is that the first five frequencies are all dipole modes; then the fifth and sixth frequencies are separated by $1.5 \Delta v_{0}$ which is theoretically quite acceptable. It does leave the problem that the amplitude modulation is not the same for all the "dipole" modes, the problem that the alternating frequency spacing then has no explanation, and the problem that the mode at $\Delta v_{0}$ above the fifth frequency is missing. But at least there is no need to explain a frequency separation of $3 / 4 \Delta v_{0}$.

This second explanation is now eliminated. The HIPPARCOS parallax for HR 1217 is $\pi=20.4 \pm 0.8$ mas; the asteroseismic parallax is $19.1 \pm 0.5$ mas if $\Delta v_{0}=68 \mu \mathrm{Hz}$; it is $\pi=11.0 \pm 0.5 \mu \mathrm{Hz}$ if $\Delta v_{0}=34 \mu \mathrm{Hz}$. The latter is ruled out. $\Delta v_{0}=68 \mu \mathrm{Hz}$ and the five frequencies are from alternating even and odd $\ell$ modes. The separation of the sixth frequency from the fifth is $3 / 4 \Delta v_{0}$; this is an ongoing mystery that can no longer be ignored in the hopes that it will go away. And what about the amplitude modulation of the even modes which are expected to be radial? That can be explained as the magnetic field modifying the surface appearance of what otherwise is an excited radial mode. Interestingly, in these proceedings that is exactly what Shibahashi \& Aerts have suggested is also the explanation for the frequency pattern seen in $\beta$ Cephei itself.

\subsection{FREQUENCY VARIABILITY IN roAp STARS}

A group from the University of Cape Town and the South African Astronomical Observatory has been observing roAp stars on a long-term basis for frequency variability for 6 years now. In this on-going project we get one hour of observation of HR 3831 on each possible night over the approximately 8 -month season when it is observable. The reason for the emphasis on this star is that it is the best-studied of the roAp stars, it has interesting rotational amplitude and phase variability which we need to remove to study the frequency variability, and the rotational variations are interesting subjects to study in their own right. We also observe for one hour once per week two other roAp stars, HD 134214 and HD 128898 ( $\alpha$ Cir). HD 134214 is singly periodic with the shortest known pulsation 
period for the roAp stars, 5.65 minutes. The very bright star $\alpha$ Cir is nearly circumpolar, and has a single large amplitude pulsation mode with only small amplitudes for other modes and small rotational sidelobes. The frequency variability of HR 3831 is discussed in Kurtz et al. (1997); we have not published O-C diagrams for the other two stars recently.

It is clear that there are variations in the pulsation cavities of these stars on time-scales of years. For HR 3831 the variations can be characterised as cyclic with a time-scale of 1.6 years. For $\alpha$ Cir the time-scale is about 6 years, and HD 134214 is harder to characterise. HR 3831 is shown in figure 4; $\alpha$ Cir and HD 134214 in figures 5 and 6. The $\mathrm{O}-\mathrm{C}$ diagrams show the pulsation phase in radians minus a constant phase. These variations cannot be easily explained as Doppler shifts caused by companions; for HR 3831 many companions would need to be hypothesised. In addition, the Ap stars have a very low incidence of binarity, only about $20 \%$ are in short period binary systems. So the frequency variations are intrinsic, and they indicate a cyclic variability in the acoustic cavity - this may be anything which affects the sound speed. One speculation we have made is that this indicates a magnetic cycle. The time scale and amplitude of the frequency variations are similar to those which are seen in the sun over the solar cycle. Magnetic fields in Ap stars are thought to be fossil, however, rather than dynamo generated, so this suggestion has not met with much approval. Whatever the physical mechanism that is at work, we have a new observational phenomenon which will eventually tell us more of the inner workings of the roAp stars via asteroseismology.

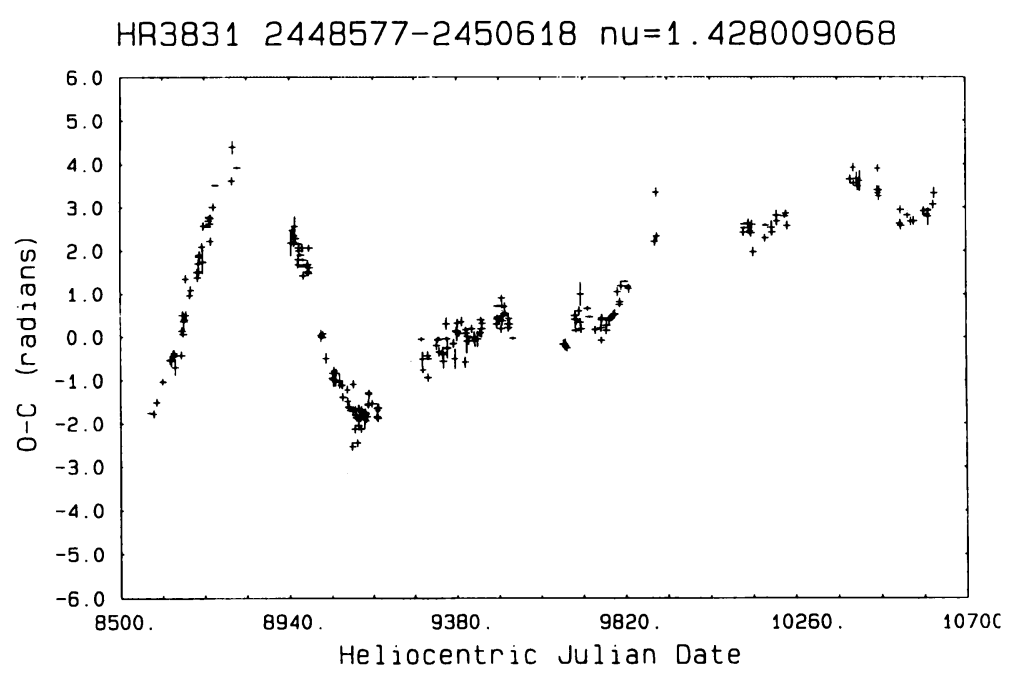

Figure 4. The phase $\mathrm{O}-\mathrm{C}$ diagram for six years of data obtained in our long-term monitoring program for HR 3831. Each point shows the difference between the leastsquares determined phase for each night of observation and the analytical rotational phase variation calculated from a model of the rotational variation. The frequency ranges over about $0.12 \mu \mathrm{Hz}$. 


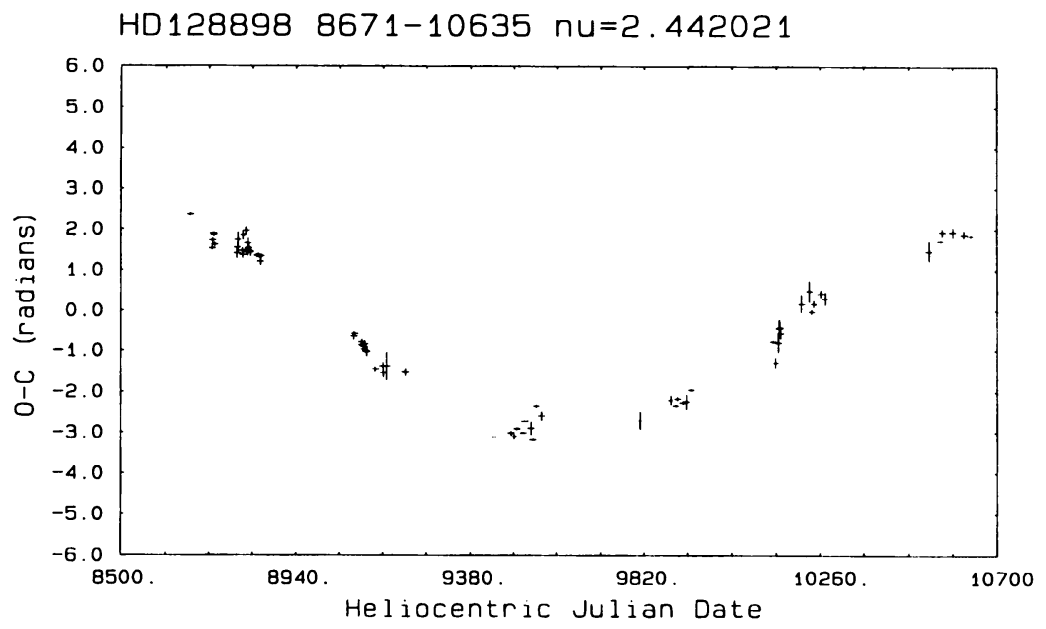

Figure 5. The phase $\mathrm{O}-\mathrm{C}$ diagram for six years of data obtained in our long-term monitoring program for $\alpha$ Cir.

HD134214 9052-10634 PHASE 0-C nu=2.94963

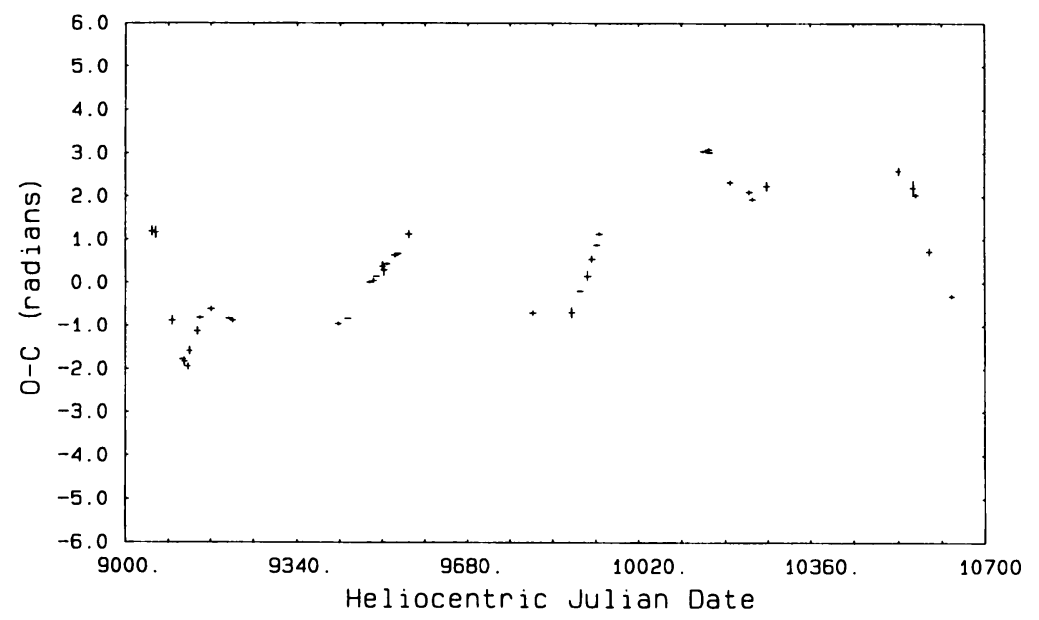

Figure 6. The phase $\mathrm{O}-\mathrm{C}$ diagram for six years of data obtained in our long-term monitoring program for HD 134214.

\subsection{ATMOSPHERIC NODES IN roAp STARS}

I was very excited by the announcement by Jaymie Matthews (Matthews et al. 1990, 1996) that atmospheric T- $\tau$ in roAp star could be determined from the amplitude of their pulsations at different wavelengths. A problem much discussed for the roAp stars is that of the 
critical frequency: Many of them seem to pulsate with frequencies so high that their vertical wavelength should be of order of the thickness of the surface boundary layer. In that case the mode should become evanescent; without very large driving there is not enough energy for modes with frequencies above the critical frequency to be maintained as standing waves. This problem is discussed in the review of Saio \& Gautschy and in the paper by Audard et al. in this volume. Matthews found that the T- $\tau$ gradient in HR 3831 was much steeper than in a normal A star model; this steepness sharpens the surface boundary and increases the critical frequency. It also results in the derivation of smaller overabundances of the rare earths and lanthanides for a given observed line strength.

Because this looked such a fruitful field, Thebe Medupe and I decided to begin a large project to observe many roAp stars in UBVRI colours to apply Matthews' technique of inferring $\mathrm{T}-\tau$ from limb darkening affects on the pulsation amplitude. Instead of modelling this first on a computer, however, we started with a simple analytic derivation of what we expected, making some reasonable first-order assumptions, such as a black body energy distribution and the Wien approximation. To our surprise we found that limb-darkening is not an important effect (Kurtz \& Medupe 1996, Medupe \& Kurtz 1997). The pulsation amplitude drops from the blue to the infrared by a factor of two greater than the drop expected for a simple black body. Limb-darkening can only account for about $12.5 \%$ of this. Much more sophisticated computer modelling confirms this result.

Medupe and I found that the observed amplitude is expected to be

$$
A_{o b s}=1.086 \frac{1}{4} \sqrt{\frac{3}{\pi}}\left(\frac{4-\mu_{\lambda}}{3-\mu_{\lambda}}\right) \cos \alpha \frac{h c}{\lambda k T_{0}} \frac{\Delta T}{T_{0}}
$$

where $\mu_{\lambda}$ is the wavelength dependent limb-darkening coefficient, $T_{0}$ is the temperature at the atmospheric level appropriate to the wavelength observed, and $\Delta \mathrm{T}$ is the semiamplitude of the pulsational polar temperature variation. What is clear is that only a strong dependence on $\Delta \mathrm{T}$ can match the observations. We suggested that we were seeing over a large fraction of the vertical wavelength of a high-overtone p-mode.

Several studies now give strong support to this suggestion. The paper by Baldry et al. in these proceedings shows radial velocity measures in which an atmospheric node is evident in $\alpha$ Cir. Pulsation phase versus equivalent width diagrams give further support in studies of $\alpha$ Cir (Baldry et al. 1997) and $\gamma$ Equ (Kanaan \& Hatzes 1997). Theoretical models are now also producing pulsation nodes in the observable atmospheres of roAp stars.

We thus have the prospect of a new asteroseismic technique for probing the atmospheres of the most peculiar stars known. Yet again, "asteroseismology will tell". 


\section{References}

Bahcall, J. N., Pinsonneault, M. H., 1992, Rev. Mod. Phys., 64, 885.

Baldry, I. K., Viskum, M., Bedding, T. R., Kjeldsen, H., Frandsen, S., 1997, MNRAS, in press.

Chaboyer, B. Demarque, P. Kernan, P. J. Krauss, L. M, 1996, Science, $271,957$.

Charpinet, S. Fontaine, G. Brassard, P. Chayer, P. Rogers, F. J. Iglesias, C. A. Dorman, B., 1997, ApJ, 483, L123.

Dziembowski W., Goode P.R., 1996, ApJ, 458, 338.

Gray, D. F., 1997, Nature, 385, 795.

Gray, D. F., Hatzes, A. P., 1997, ApJ, in press.

Hansen, C. J., Kawaler, S. D., 1991, Stellar Interiors: Physical Principles, Structure and Evolution, Springer-Verlag, New York.

Kanaan, A., Hatzes, A. P., 1997, preprint.

Kilkenny, D., Koen, C., O’Donoghue, D., Stobie, R. S., 1997, MNRAS, 285, 640.

Koen, C., Kilkenny, D., O’Donoghue, D., van Wyk, F., Stobie, R. S., 1997, MNRAS, 285, 645.

Kurtz D.W., Garrison, R. F., Koen, C., Hofmann, G. F., Viranna, N. B., 1995, MNRAS, 276, 199.

Kurtz D.W., Matthews J.M., Martinez P., Seeman J., Cropper M., Clemens J.C., Kreidl T.J., Sterken C., Schneider H., Weiss W.W., Kawaler S.D., Kepler S.O., van der Peet A., Sullivan D. J., and Wood H.J., 1989, MNRAS, 240, 881.

Kurtz D.W., Sullivan D. J., Martinez P., Tripe P., 1994, MNRAS, 270, 674.

Kurtz D.W., van Wyk F., Roberts, G., Marang F., Handler, G., Medupe, R., Kilkenny, D., 1997, MNRAS, 287, 69.

Kurtz, D. W., Medupe, R., 1996, Bull, Ast. Soc. India, 24, 291.

Marcy, G. W., Butler, P. R., Williams, E., Bildsten, L., Graham, J. R., Ghez, A. M., Jernigan, J. G., ApJ, $481,926$.

Matthews J.M., Wehlau W.H., Rice, J., Walker G.A.H., 1996, ApJ, 459, 278.

Matthews J.M., Wehlau W.H., Walker G.A.H., 1990, ApJ, 365, L81.

Matthews, J. M., Kurtz, D. W., Martinez, P., 1997, in preparation.

Mayor, M, Queloz, D., 1995, Nature, 378, 355.

Medupe, R., Kurtz, D. W., 1997, MNRAS, submitted.

O’Donoghue, D., Lynas-Gray, A. E., Kilkenny, D., Stobie, R. S., Koen, 1997, MNRAS, 285, 657.

Richard, G., Vauclair, S., Charbonnel, C., Dziembowski, W. A., 1996, A\&A, 312, 1000.

Shibahashi H., Saio H., 1985, PASJ, 37, 245.

Shibahashi H., Takata M., 1993, PASJ, 45, 617

Stobie, R. S., Kawaler, S. D., Kilkenny, D., O’Donoghue, D., Koen, 1997, MNRAS, 285, 651.

Takata M., Shibahashi H., 1995, PASJ, 47, 219.

Unno W., Osaki Y., Ando H., Saio H., Shibahashi H., 1989, Non-radial oscillations of stars, Univ. Tokyo Press. 